\title{
Research on Flexible Business Process of Bank Modeling Based on EPC
}

\author{
Wang Xia, Man Junpeng and Liu Lan \\ School of information management, \\ Shanghai Finance University, Shang Hai, China \\ wangx@shfc.edu.cn,1602760398@qq.com,liul@shfc.edu.cn
}

\begin{abstract}
As the further development of banking business for personalization and diversification, the bank information system is capable of dynamically adjusting business process and has set higher requirements for the flexible mechanism. This paper constructs a model of the business process based on Event-driven Process Chains (EPC), puts forward the concrete specification in the process of construction. A case study was implemented to validate the model using Pi-calculus. The results show that this model can be a powerful tool for the creation of flexible or customized business process.
\end{abstract}

Keywords: Business process modeling; EPC; Construction algorithm; Pi-calculus

\section{Introduction}

With the intense competition in the banking sector, financial products are continuously developing. The existing process model is facing tough challenges. The users of business process have been changed. Not only has it referred to the huge amount of ordinary users but also the personalized demands. The banks are required to complete the business process reengineering under risk and time constraint, which are imposed on frequently updated demand. At present, the business process design of domestic banking is mainly based on the traditional hierarchy structure which has many defects such as the complexity of information review, the lack of coordination among departments and so on. In the aspect of information system development, the construction of data centralization and all kinds of business system is completed. But a multitude of problems exist about the lack of flexibility of business process. They are mainly due to the independent among business functions, be in capable of data information exchange.

In recent years, it is becoming a research hotspot on how to make the process assembly of business system more flexible, agile. The FIDO [1] modeling approach proposed an architecture based on the agent technology. It supports the perception, interpretation, prediction, automation and the response for the changes of business processes. According to the BRADE [2] method, a business process model is divided into three sub models according to the life cycle. But the hardest part is to determine what detail information a sub model should contain. IBM proposed an artifact-centric business process modeling ACOM (Artifact-centered Operational Modeling) [3] which organically combines the data and process management. According to the paper [4], it proposes a kind of specification language ABSL which is based on CTL (Computation Tree Logic). It is used to specify the life cycle behaviors of artifact in the artifact-centric model. SynchroFlow [5] used the management and integration system of business process which is oriented to the business domain to implement the dynamic management of business processes. Through the independent encapsulation of information exchange mechanism for business processes, functions and participants, the business logic, business data and business operation entity are loosely coupled. As it can be seen from the modeling method discussed above, a number of them are the static requirements modeling for the business process, data, 
function and behavior. The adjustment of business process can be achieved through the flexibility of IT technology. But it is lack of the modeling and analysis of the process properties which are reflected by the dependence, relevance, restriction between business functions.

During the applications of banking business, when the business process changes a little, it can meet the personal requirements in certain level through the process adjustment due to the flexible mechanism of modeling software. But due to the lack of dependence modeling on inter-level of business processes, when the great changes of the business process requirements have taken place, the business software should be reanalyzed, designed and implemented. It not only cannot cope with the rapidly changing of market environment, but also greatly increased the cost.

The paper describes the business process modeling based on the variability analysis of bank business process. The flexible bank business process model is constructed based on EPC and is verified. So that it can support the improvement of the banks' ability that is to agile response to the markets and the changes of customer requirements.

\section{Business Process Modeling Descriptions}

\subsection{EPC Modeling Principle}

EPC is a kind of graphical business process modeling language proposed by Keller et $a l$ in the 1990's. It is used to describe of the business process. The basic idea of EPC is that events trigger functions, or the executed functions cause events. Its features are its intuitive nature and simplicity of modelling as well as its comprehensibility and easy interpretation [6]. The $\mathrm{E}$ is a finite set of events. The $\mathrm{P}$ is a finite set of process. The $\mathrm{C}$ is a finite set of logical connection in process chain. The $\mathrm{T}$ is a function mapping that each connection in process chain is mapped to the elements in $\{\mathrm{AND}, \mathrm{Or}, \mathrm{XOR}\}$, namely $\mathrm{T} \in$ $\mathrm{C}-->\{\mathrm{AND}, \mathrm{OR}, \mathrm{XOR}\}$. Relation $\mathrm{A}$ is a set of directional connective arcs in the process chain [7].

The typical elements of the EPC are event, function and connector (such as AND, OR, XOR). EVENT is a kind of status of the environment. It can trigger the functions that the arc points to. And the arc is from it. FUNCTION is triggered by EVENT which completes specific tasks or actions. CONNECTOR includes three connectors: AND, OR, XOR. Each CONNECTOR can be divided into the join type and split type, so there are total 6 kinds of CONNECTOR: AND-join, AND-split, OR-join, OR-split, XOR-join, XOR-split.

\subsection{Bank Business Process Description}

Because of the particularity of financial industry, bank business process must be reasonable and safe. All these features cannot be changed by the modification for the bank business process. Or even a little leak will cause huge economic losses. In order to avoid potential safety hazard brought by adjusting or customizing the business process, the changes of business process must meet the following conditions: (see, Figure 1)

- The main functions of the original process cannot be deleted. The so-called main function is a required function of the process. As shown in Figure 1, there are six types of main function such as client application, processing application, approval, signing contracts, loans, repayment of the loan. All of them cannot be deleted.

- Because a functional modifications may be related to the modifications of many other functions. Therefore, as a matter of principle, the priority is little change. 


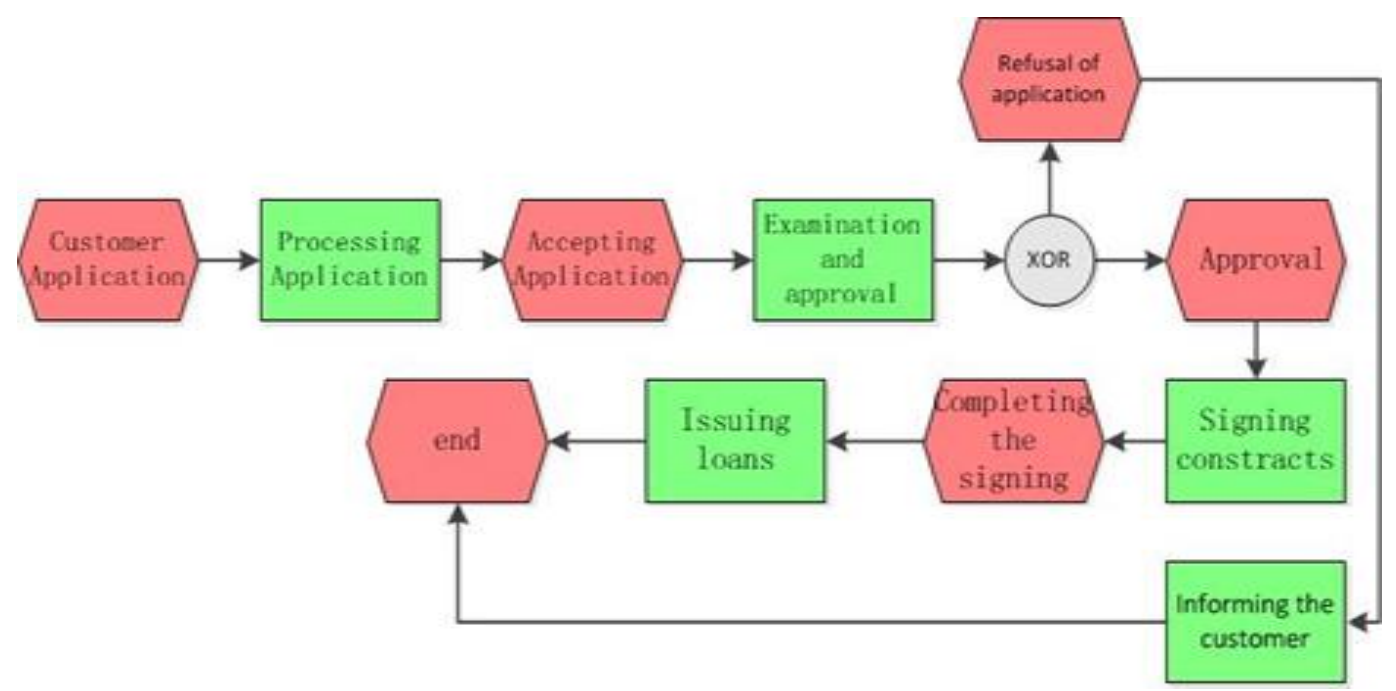

Figure 1. EPC Modeling of Business Process for Bank Loan

\section{Flexible Business Process Modeling of Bank}

EPC model of the initial business process is built according to the input requirements. When the business process needs to be changed, the transformer will call the EPC model and rebuild the business process. The constructor is to save the changes to the current EPC model, and then call the model generator. The generator will execute the construction algorithm of business process and output the complete business processes to the current EPC model. Finally, the transformer calls the validator. It validates whether the process meets the expected requirement through the PI - calculus of process modeling and execution (see, Figure 2).

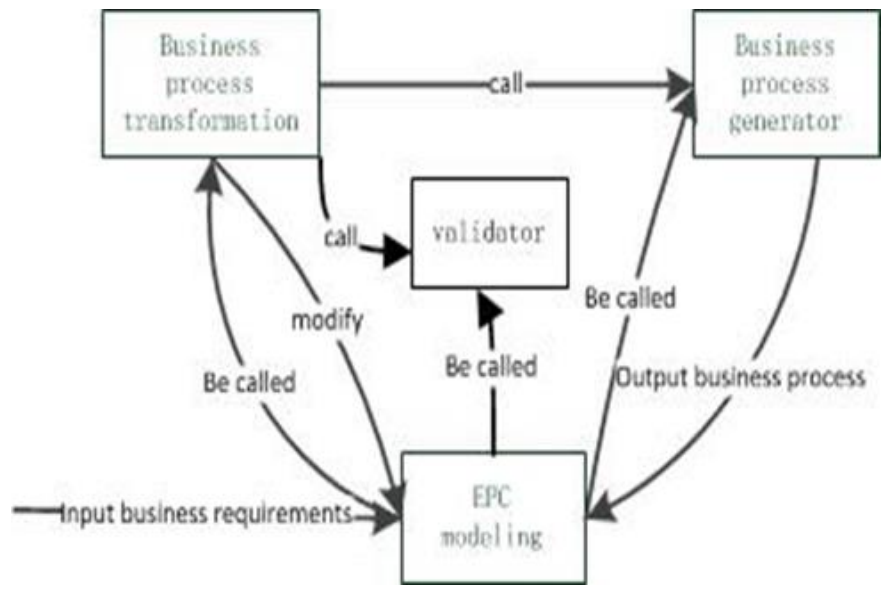

Figure 2. Architecture for the Flexible Business Process Model

The model is based on the EPC. On the one hand, it can replace the specific function of EPC model. On the other hand, it can replace a child EPC model. It is able to realize the replacement of function as well as the process. In short, the model could make the business process more flexible according to the requirements.

\subsection{The Basic Idea}

First, the experts of bank process build model for the main functions of business process, or the EPC model for the top business process. Then, they build EPC models for each lower layer which should be marked to replace the function of the upper EPC model. 
So according to the design ideas of top-down and layer decomposition, the EPC model is built. There are the following requirements in the process of establishing EPC model:

- If there are multiple input / output events in any EPC model, these events don't exist any logical relationship (i.e., any event may not be replaced by the other events or their logical calculus). And the corresponding functions of them in the upper EPC model have the same number and equivalent input / output events. For example, there are two output events for the loan approval function as shown in Figure 3. It is obviously needed that there are two corresponding output events in Figure 1.

- When the functions and events appear alternately, the functions must be directly connected to the corresponding events in addition to using the XOR-split and the OR-split connector which can be indirectly connected (For example in Figure 3, the three functions and the corresponding events which are connected to the XOR -split). By default the connection between $\mathrm{A}$ and $\mathrm{B}$ uses directed line which is from $\mathrm{A}$ to connect $\mathrm{B}$. the same below.

In addition, the revised process will be verified by using the formal language such as Pi-calculus. It is mainly verity the rationality of the transformation process.

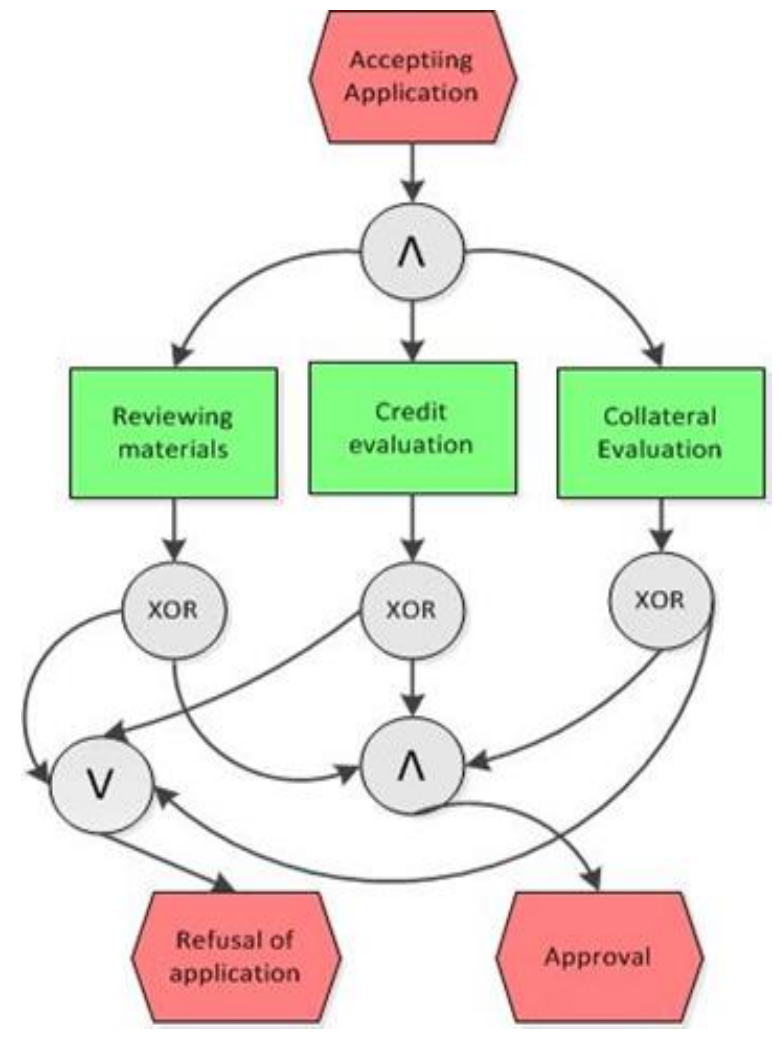

Figure 3. EPC Modeling of the Bank Loan Approval

Specially, there are four basic replacement algorithms in the EPC model that could satisfy the above requirements.

- Case 1: the replaced function with only one output event as shown in Figure 4. The upper left part is the EPC model. The right part is the second layer of EPC model and describes the execution process of $\mathrm{F} 1$ that is in the left part. In the process of replacement, the content surrounded with dotted line will be replaced by the content surrounded with solid line (the same below) 


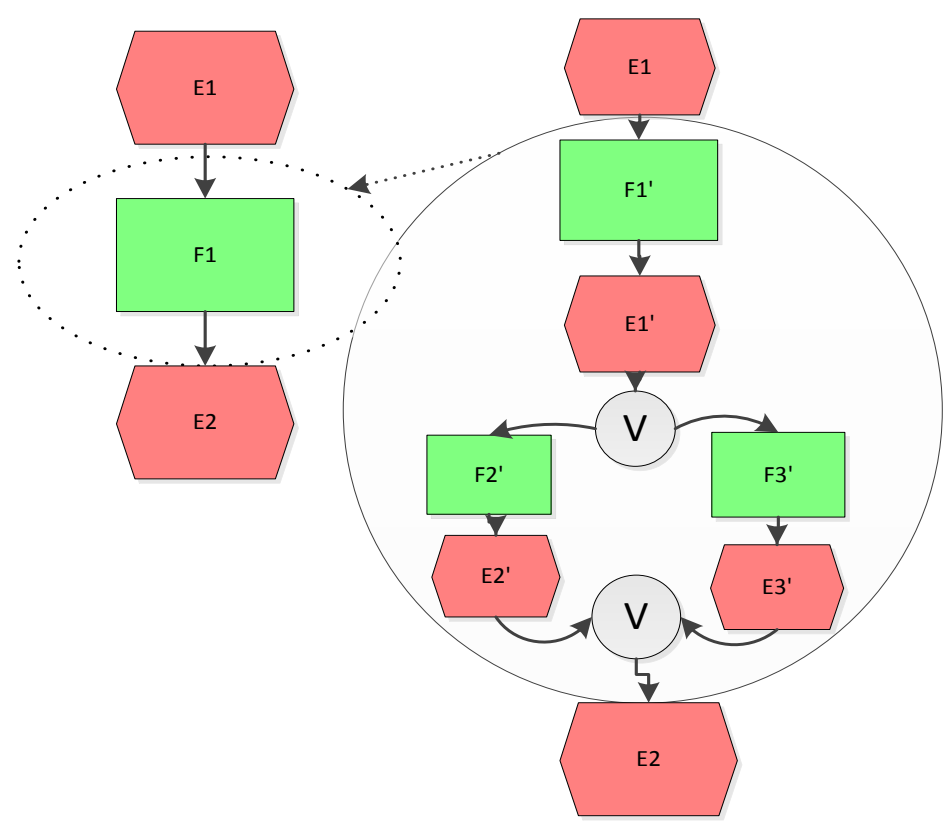

Figure 4. The Replacement of Case 1

- Case 2: the replaced function directly connects with the XOR-split or the OR-split connector, and then indirectly connects with other events as shown in Figure 5.

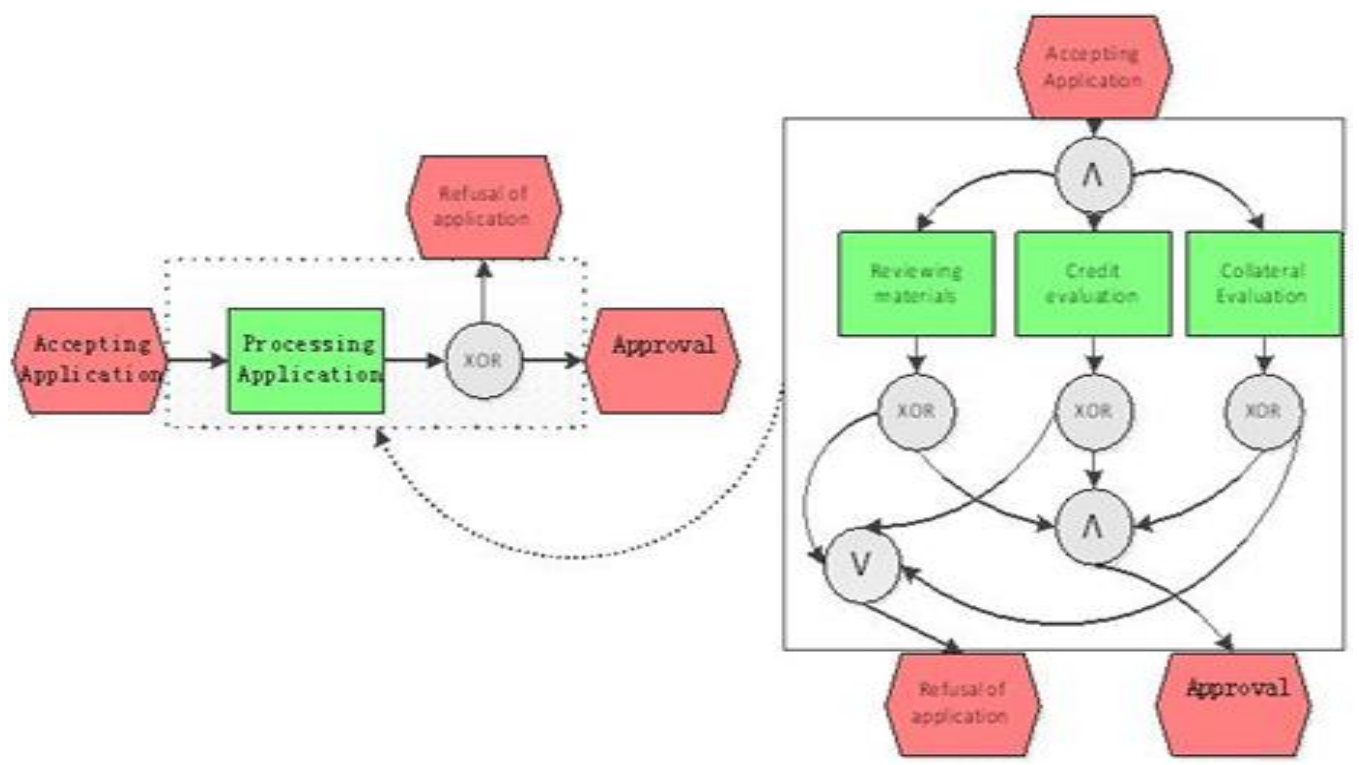

Figure 5. The Replacement of Case 2

Figure 6.

- Case 3: the replaced function connects with one of the join connector as shown in

- Case 4: the replaced function connects with one of the split connector as shown in Figure 7.

The other cases can be deduced by the above four cases. In fact, the replacement in Figure 7 is the combination of case 4 and case 2 . 


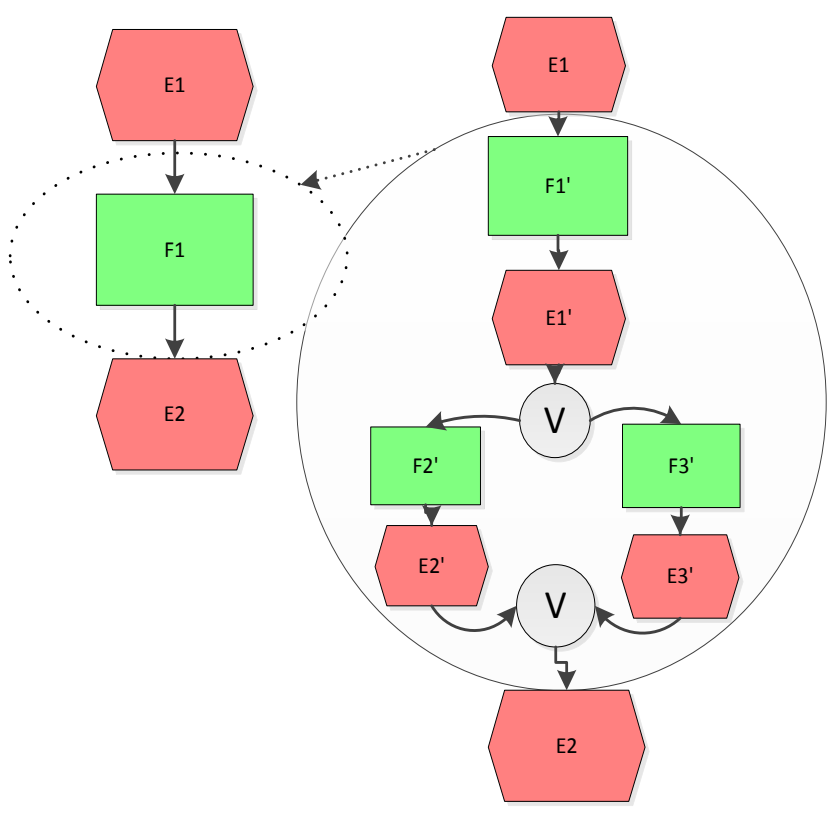

Figure 6. The Replacement of Case 3

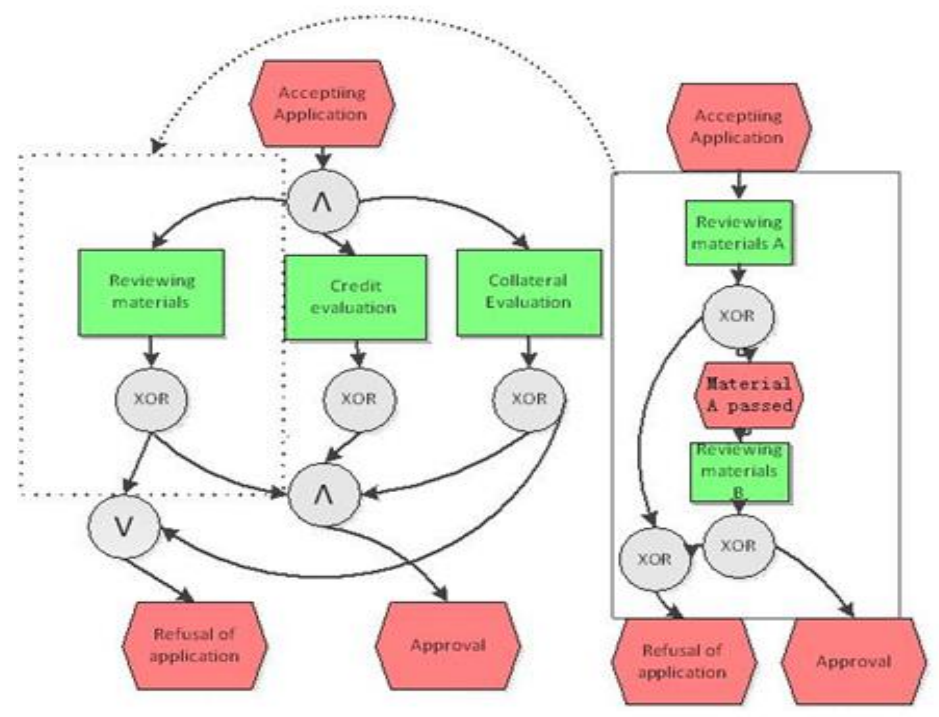

Figure 7. The Replacement of Case 4

\subsection{Business Process Construction Algorithm}

The paper proposed the algorithm of the function substitution to construct the whole process referring to the combination algorithm of the scenarios to the process which was presented in [8]. After the business process generator reads the current EPC model, it will call the following algorithm to construct the business process.

Procedure function-replace (EPC EP1, Function F1, EPC EP2) \{

// EP2 defines the detailed process of the function F1 in EP1

Define the antecedent event of $\mathrm{F} 1$ as Ein1, $\cdots$, Einm $(1 \leq \mathrm{m})$ consequent event as Eout $1, \cdots$, Eoutn $(1 \leq \mathrm{n})$, 
Replace the parts of between every $\operatorname{Eini}(1 \leq \mathrm{i} \leq \mathrm{m})$ and Eoutj $(1 \leq \mathrm{j} \leq \mathrm{n})$ in EP1 with the parts of between every $\operatorname{Eini}(1 \leq \mathrm{i} \leq \mathrm{m})$ and Eoutj $(1 \leq \mathrm{j} \leq \mathrm{n})$ in EP2

$\forall$ Function or connector or Eini $(1 \leqslant \mathrm{i} \leqslant \mathrm{m}$ ) (Unity is denoted as A in EP2

If A directly connects with an element of EPC model (denoted as B) in EP2, A connects with element B which has the same name and type in EP1.

If $\mathrm{f}$ A directly connects with an element of EPC model (denoted as B) in EP1, A connects with element B which has the same name and type in EP2.

\}

The construction algorithm of business processes derives from the functional replacement algorithm, where EPC1 is the top of the EPC model:

Procedure DFS (EPC EP1) \{

For each (Function F in EP1) \{

If (exists EPC model EP2 mark the replace function F) \{

If (EP2 does not marked)\{

DFS (EP2);

Mark EP2;

\}

Function-replace (EP1, F, EP2);

//replace F with the EP2 using the replacement algorithm and form a new EP1;

\}

\}

Procedure Constructor (EPC EPC1) \{

Mark EPC1

DFS (EPC1);

\}

\subsection{Implementation}

Each function is compatible in the initial EPC model of business process which is constructed according to the business requirements. That is to say, the processing mechanisms in the other functions are set for the data or information which is transferred from each function to them.

The scope of data or information (denoted as the range of the function) generated by $\forall$ $\mathrm{F} 1$ is $\mathrm{R} 1$. If in the implementation process $\mathrm{F} 2$ will use the data or information which are generated in F1 and the scope of input information that can be handle by F2 (denoted as the definition domain of the function) $\mathrm{D} 2$, there is $\mathrm{R} 1 \subseteq \mathrm{D} 2$. This quality is the important factor which assures the process can be executed correctly according to the design intention.

All changes to the process cannot destroy the nature, otherwise the transformation of the process is invalid or will cause the process execution stops or may produce error results.

The transformation of the process which is executed by the transformer is divided into two types. The first is the transformation of functions in the processes. The second is the transformation of the execution order between functions in the process. According to the different treatment, the transformations of functions are classified into three types:

Type A: adding a function in front of a certain function;

Type B: adding a function after the output event;

Type C: Modifying the execution mechanism of certain function.

For the type B, it is easy to be handled. It only needs to directly add the corresponding function and subsequent events after the output event and does not need to transform the original function. The paper focuses on the function transformation of type A and type C. 
As shown in figure 8 for type A:

Under the condition of without changing F1

Adding functions F2 in front of the F1,

Denoting F1's definition domain as D1, F2's definition domain as D2, F2's range as R2, then there must be $\mathrm{D} 1 \subseteq \mathrm{D} 1$ and $\mathrm{R} 2 \subseteq \mathrm{D} 1$.

If the above conditions are met, the EPC model is constructed to replace the F1.

Then $\mathrm{F} 1$ is replaced in accordance with the replacement algorithm.

If the above conditions are not met, adding $\mathrm{F} 2$ is equivalent to the transformation of $\mathrm{F} 1$ according to the type $\mathrm{C}$. The modified $\mathrm{F} 1$ (denoted as F1') is equivalent to the function that after combining F1 and F2.

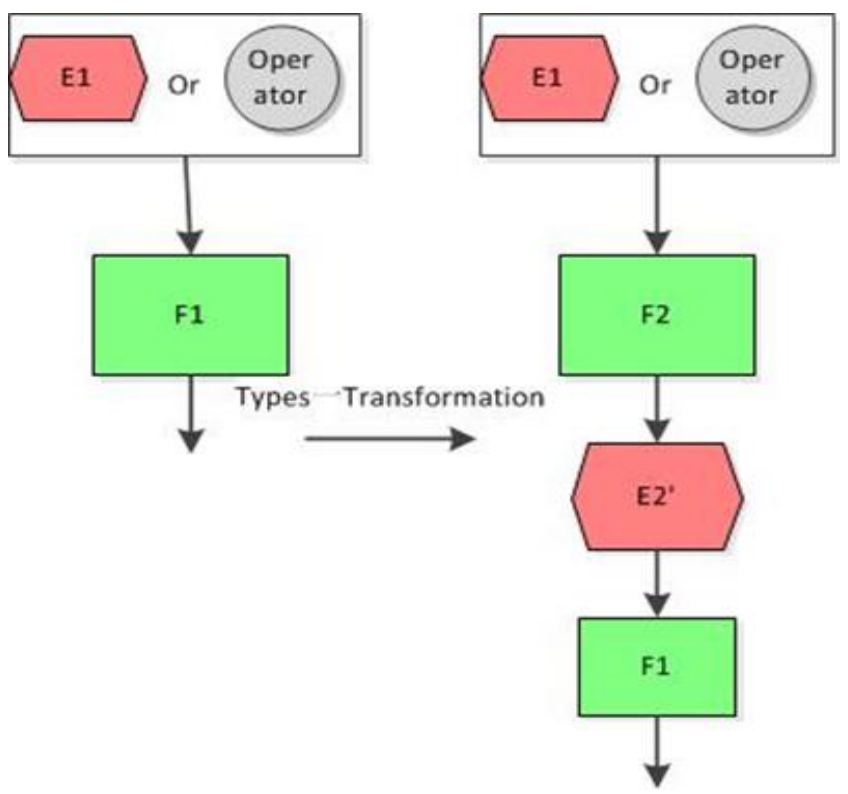

Figure 8. Type A Diagram of Function Transformation

The transformation of type C:

Transforming F1 into F1'

If $\mathrm{D} 1 \subseteq \mathrm{D} 1$ '（denoted as condition 1) and $\mathrm{R} 1$ ' $\subseteq \mathrm{R} 1$ (denoted as condition 2) then end;

Or, if the condition 1 is not available, then redesigning F1', or (condition 1 is available but condition 2 is not),

Then Redesigning F1'and meeting condition 1 and condition 2

Or according to type $\mathrm{C}$, the data or information functions (denoted as Fset) generated by F1 is transformed in the top EPC model which constructed through using function replacement algorithm.

If $\forall F \in F$ set then $R 1^{\prime}-F \subseteq D F$ ( $R 1$ ' $-F$ is the set of values which $F 1$ ' outputs to $F$, DF is the definition domain.),

Then end.

The process of transformation of the type $\mathrm{C}$ is defined recursively, and different from the depth algorithm. A function may still need improvement after the transformation. So the cost of type B is very high.

In addition, the transformation of deleting some functions from the business process model also belongs to the type C. A function is removed which is equivalent to its transformation into an adapter. It makes the functions which are on both sides of deleted functions run properly. They cannot connect together due to the mismatch of the interface before. A function can be deleted is equivalent to an ability to find an adapter, making the 
revised process not destroy the compatible properties between the various functional process.

There are two aspects of the transformation of business process execution order: The first is EPC redesigned. The second is transformation for all related function. So the transformation of the execution order of function can be achieved by using the function transformation algorithm.

\section{Business Process Model Validation}

After the modeling for the transformation of business process, the transformer will call the validator to check the rationality. The validator generates the code for the Pi-calculus according to the process.

Pi-calculus [9] is found by Robin Milner et al through extending CCS (calculus of Communicating Systems). It is a kind of model that is used to describe and analyze concurrent system, and it has become one of the most important models of concurrency theory. It inherited concise semantic theory that is mutual simulation from CCS. It is easy to verify the correctness of process modeling by means of the theory [10].

There are two types of entities in the Pi- calculus. One is Name. The other is Process. Data, channel, variables, parameters are the types of Name.

Process expression of Pi-calculus can be described in the following equation (1):

$$
\begin{aligned}
& \mathrm{P}::=\mathrm{M}|\mathrm{P} 1| \mathrm{P} 2|\mathrm{vzP}| ! \mathrm{P} \\
& \mathrm{M}::=0|\pi . \mathrm{P}| \mathrm{M} 1+\mathrm{M} 2 \\
& \pi:=^{-} \mathrm{x}<\mathrm{y}>|\mathrm{x}(\mathrm{z})| \tau \mid[\mathrm{x}=\mathrm{y}] \pi
\end{aligned}
$$

- $\mathrm{P}$ expression for the process

- $\mathrm{P} 1 \mid \mathrm{P} 2$ expression for the concurrent execution of process $\mathrm{P} 1$ and process $\mathrm{P} 2$

- vzP expression that Name $\mathrm{Z}$ is the internal name of process $\mathrm{P}$

- $! \mathrm{P}=\mathrm{P} \mid ! \mathrm{P}$ expression that process $\mathrm{P}$ can be replicated infinitely.

- $\bar{x}<y>$ expression for receiving $\mathrm{y}$ through channel $\mathrm{x}$, expression for sending $\mathrm{y}$ through channel $\mathrm{x}$ 。

- $[x=y] \pi$ expression for executing action $\pi$ if $\mathrm{x}$ is equal to $\mathrm{y}$.

Using the Pi- calculus to describe elements in EPC [11-12], the first 6 connectors are described:

AND-join:

$$
C_{N j}=\mathrm{a}(\mathrm{x}) \cdot \mathrm{b}(\mathrm{y}) \cdot \tau \cdot \overline{\mathrm{c}}<\mathrm{z}>C_{N j}^{\prime}
$$

AND-split:

$$
\mathrm{C}_{\text {Ns }}=\mathrm{z}(\mathrm{x}) \cdot \tau \cdot(\overline{\mathrm{b}}<\mathrm{x}>.0 \mid \overline{\mathrm{c}}<\mathrm{x}>.0)
$$

OR-join:

OR-split:

$$
\begin{aligned}
\mathrm{C}_{\mathrm{vj}} & =\mathrm{a}(\mathrm{x}) \cdot \tau \cdot \overline{\mathrm{c}}<\mathrm{x}>\cdot \mathrm{C}_{\mathrm{vj}}^{\prime}+\mathrm{b}(\mathrm{y}) \cdot \tau \cdot \overline{\mathrm{c}} \\
& <\mathrm{y}>\cdot \mathrm{C}_{\mathrm{vj}}^{\prime}+\mathrm{a}(\mathrm{x}) \cdot \mathrm{b}(\mathrm{y}) \cdot \tau \cdot \overline{\mathrm{c}}<\mathrm{z}>\mathrm{C}_{\mathrm{vj}}^{\prime}
\end{aligned}
$$

XOR-join:

XOR-split:

$$
\begin{aligned}
& \mathrm{C}_{\mathrm{Vs}}=(\mathrm{vexec}, \mathrm{msg}) \tau .\left(\mathrm{A}_{1} \mid \mathrm{A}_{2}\right) \\
& \mathrm{A}_{1}=\mathrm{c}(\mathrm{x}) .(\mathrm{exec}<\mathrm{a}>. \overline{\mathrm{msg}}<\mathrm{x}>.0+\overline{\operatorname{exec}} \\
&<\mathrm{b} . \overline{\mathrm{msg}}<\mathrm{x}>.0+\overline{\operatorname{exec}}<a>. \overline{m s g}<x>. \overline{\operatorname{exec}}<b>. \overline{m s g}<x>.0) \\
& \quad \mathrm{A}_{2}=\text { !exec(ch).msg(x). } \overline{\mathrm{ch}}<\mathrm{x}>.0
\end{aligned}
$$

For the Event,

$$
C_{x j}=a(x) \cdot \tau \cdot \bar{c}<x>C_{x j}^{t}
$$

$$
\mathrm{C}_{\mathrm{Xs}}=c(\mathrm{x}) \cdot \tau \cdot\left(\overline{\mathrm{a}}<\mathrm{x}>\cdot \tau \cdot \mathrm{C}_{\mathrm{Xs}}^{\prime}+\overline{\mathrm{b}}<\mathrm{x}>\text {. } \tau \cdot \mathrm{C}_{\mathrm{Xs}}^{\prime}\right)
$$

For the Function, 


$$
F=a(x) \cdot \tau \cdot \bar{b}<y>.0
$$

The bank loan approval process modeling is shown (see, figure 9) using these expressions:

Accepting the application Event:

$E 1=\bar{a}<x>.0$

AND-split connector :

$C 1=a(x) \cdot(\bar{b}<x>|\bar{c}<x>| \bar{d}<x>)$

Reviewing materials function:

$F 1=b(x) \cdot \tau \cdot e<y>.0$

Credit evaluation function:

$F 2=c(x) \cdot \tau \cdot \bar{f}<z>.0$

Collateral evaluation function:

$F 3=d(x) \cdot \tau \cdot \bar{g}<r>.0$

Three XOR-split connectors :

$C 2=e(y) \cdot \tau \cdot(\bar{h}<y>+\bar{l}<y>)$
$C 3=f(z) \cdot \tau \cdot(\bar{J}<z>+\bar{k}<z>)$
$C 4=g(r) \cdot \tau \cdot(\bar{l}<r>+\bar{m}<r>)$

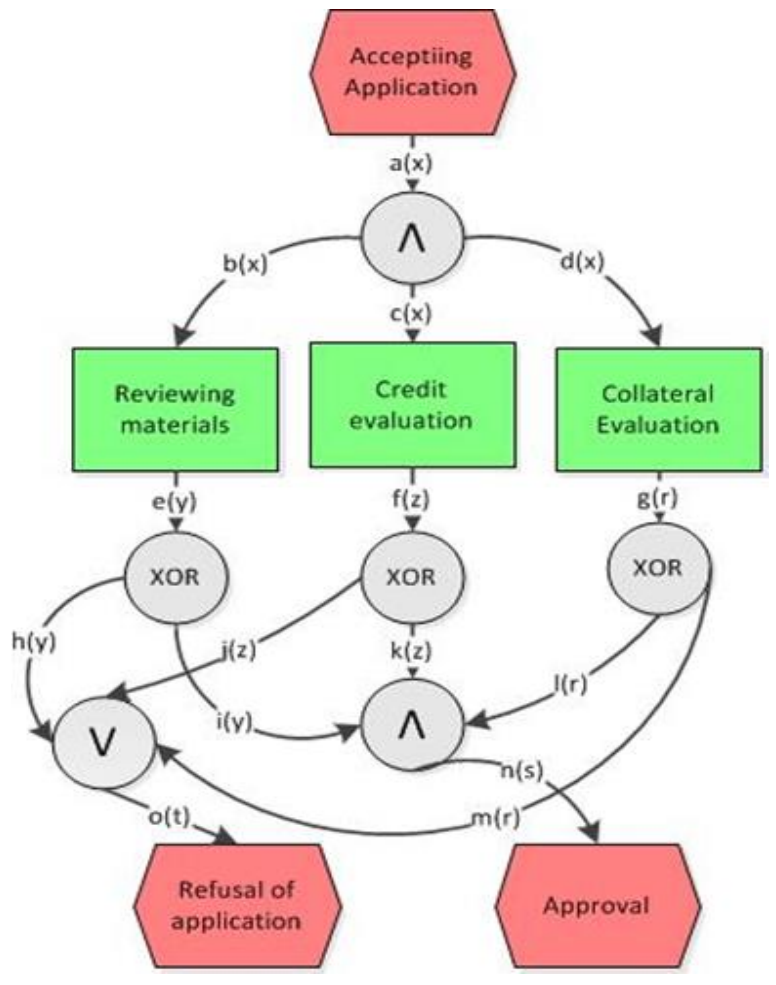

Figure 9. Pi-Calculus Description of EPC Shown in Figure 3

OR-join connector :

$C 5=(h(y) \cdot \tau \cdot \bar{o}<t>.0+j(z) \cdot \tau \cdot \bar{o}<t>.0+m(r) \cdot \tau \cdot \bar{o}<t>.0$

$+h(y) \cdot j(z) \cdot \tau \cdot \bar{o}<t>.0+h(y) \cdot m(r) \cdot \tau \cdot \bar{o}<t>.0$

$+j(z) \cdot m(r) \cdot \tau \cdot \bar{o}<t>.0+h(y) \cdot j(z) \cdot m(r) \cdot \tau \cdot \bar{o}<t>.0$

AND-join connector :

$C 6=(i(y) \cdot k(z) \cdot l(r)) \cdot \tau, \bar{n}<s>.0$

Refusal of application event:

$E 2=o(t) \cdot 0$

$E 3=n(s) .0$

Then: 
$E P C_{2}=E 1|E 2| E 3|C 1| C 2|C 3| C 4|C 5| C 6|F 1| F 2 \mid F 3$

In accordance with the relevant rules of Pi- calculus [9], the evolution of EPC2 is shown below:

$$
\begin{aligned}
& E P C_{2} \stackrel{a}{\longrightarrow} 0|E 2| E 3|\bar{b}<x>| \bar{c}<x>|\bar{d}<x>| C 2|C 3| C 4|C 5| C 6|F 1| F 2 \mid F 3 \\
& \stackrel{b}{\longrightarrow} 0|E 2| E 3|0| \bar{c}<x>|\bar{d}<x>| C 2|C 3| C 4|C 5| C 6|\tau . \bar{e}<y>.0| F 2 \mid F 3 \\
& \stackrel{d}{\longrightarrow} 0|E 2| E 3|0| \bar{c}<x>0|C 2| C 3|C 4| C 5|C 6| \tau . \bar{e}<y>.0|F 2| \tau . \bar{g}<r>.0 \\
& \stackrel{c}{\longrightarrow} 0|E 2| E 3|0| 0|0| C 2|C 3| C 4|C 5| C 6|\tau . \bar{e}<y>.0| \tau . \bar{f}<z>.0 \mid \tau . \bar{g}<r>.0 \\
& \stackrel{\tau}{\longrightarrow} 0|E 2| E 3|0| 0|0| C 2|C 3| C 4|C 5| C 6|\tau . \bar{e}<y>.0| \bar{f}<z>.0 \mid \tau . \bar{g}<r>.0 \\
& \stackrel{\tau}{\longrightarrow} 0|E 2| E 3|0| 0|0| C 2|C 3| C 4|C 5| C 6|\bar{e}<y>.0| \bar{f}<z>.0 \mid \tau . \bar{g}<r>.0 \\
& \stackrel{\tau}{\longrightarrow} 0|E 2| E 3|0| 0|0| C 2|C 3| C 4|C 5| C 6|\bar{e}<y>.0| \bar{f}<z>.0 \mid \bar{g}<r>.0 \\
& \stackrel{f}{\longrightarrow} 0|E 2| E 3|0| 0|0| C 2|\tau .(\bar{j}<z>+\bar{k}<z>)| C 4|C 5| C 6|\bar{e}<y>.0| 0 \mid \bar{g}<r>.0 \\
& \stackrel{g}{\longrightarrow} 0|E 2| E 3|0| 0|0| C 2|\tau \cdot(\bar{j}<z>+\bar{k}<z>)| \tau \cdot(\bar{l}<r>+\bar{m}<r>)|C 5| C 6|\bar{e}<y>.0| 0 \mid 0 \\
& \stackrel{e}{\longrightarrow} 0|E 2| E 3|0| 0|0| \tau \cdot(\bar{h}<y>+\bar{i}<y>)|\tau \cdot(\bar{j}<z>+\bar{k}<z>)| \tau \cdot(\bar{l}<r>+\bar{m}<r>)|C 5| C 6|0| 0 \mid 0 \\
& \stackrel{\tau}{\longrightarrow} \stackrel{\tau}{\longrightarrow} \stackrel{\tau}{\longrightarrow} 0|E 2| E 3|0| 0|0|(\bar{h}<y>+\bar{i}<y>)|(\bar{j}<z>+\bar{k}<z>)|(\bar{l}<r>+\bar{m}<r>)|C 5| C 6|0| 0 \mid 0 \\
& \stackrel{h}{\longrightarrow} \stackrel{k}{\longrightarrow} \stackrel{l}{\longrightarrow} 0|E 2| E 3|0| 0|0| 0|0| 0|\bar{o}<t>.0| i(y) . k(z) . l(r) . \tau . \bar{n}<s>.0|0| 0 \mid 0 \\
& \stackrel{o}{\longrightarrow} 0|0| E 3|0| 0|0| 0|0| 0|0| i(y) \cdot k(z) \cdot l(r) \cdot \tau \cdot \bar{n}<s>.0|0| 0 \mid 0
\end{aligned}
$$

The evolution represents that reviewing material does not pass eventually and leads to refusing the application.

If the definition of $\mathrm{C} 2$ as follows:

$$
C 2=e\left(y^{\prime}\right)\left(\sum_{y \in D_{C 2}}\left[y^{\prime}=y\right] \cdot \tau \cdot\left(\bar{h}<y^{\prime}>+\bar{l}<y^{x}>\right)\right)
$$

Wherein $\mathrm{y}^{\prime}$ for the received data, Dc2 for the information set that $\mathrm{C} 2$ can process, and $\sum_{i} \pi_{i} P_{i}$ is equivalent to $\pi_{1} \cdot \mathrm{P}_{1}+\pi_{2} \cdot \mathrm{P}_{2}+\cdots \cdots$

According to the definition, $\mathrm{C} 2$ can continue to response only when it can process the received data, otherwise it will always be blocked. Using this definition, C2 can effectively prevent leading to failure due to the wrong modification of the input function of $\mathrm{C} 2$ (or event). Meanwhile, it can be used to validate the transformation of bank business process based on the blocking properties. Event and Function can also be defined in this way.

In addition, web service substitution verification was discussed in the reference [13]. The correctness of Web service composition model and whether it meets the design requirements were studied in the references [14-15]. All of them can be applied in the validation of bank process transformation. Methods given in the references [13-15] can be used to do further analysis for the rationality and validity of the functional replacement.

PIEFP, VPAM, JACK toolkit and MWB (Mobility Workbench), a set of tools to support Pi- calculus can be used to do auxiliary validations. For example, if the validation is done by the MWB tool, it can be verified whether the model meets the design expectations through simulating interactive operations of processes by 'step' command. The MWB tool is constructed by using SML. It can detect and remove some of common errors by using the MWB tool, and improve the efficiency and accuracy of the validation. In addition to the 'step', MWB also provides the 'deadlocks' and 'weq' commands for deadlock detection and verification the rationality of function replacement [12].

\section{Conclusion}

With the rapid development of global economic integration, customer needs have become more diversified, efficient and personalized. For this reason, it can be achieved all 
of the above functionality without the need to refactor codes. The paper proposes a kind of model to improve the business process of flexible mechanism. It is based on EPC to the modeling of the business process. In addition, the paper builds a particular instance of the bank business process and is verified by the PI calculus.

Based on the analysis of the characteristics of bank business process, the paper provides the flexible bank business process model based on EPC, optimizes the process construction algorithm, and discusses various implementation process of replacement. The study on the customization technology of bank business process is a preliminary exploration, and there are still many further researches. It has a certain reference value to study the flexible bank business customization that the function replacement modeling and the thought of validation on the transformation model.

\section{Acknowledgements}

Research Base of Shanghai Humane Innovations, He Ying Research Studio, Institute for Development Strategic Studies of Shanghai.

\section{References}

[1] L. Shunk, J. kim, and H. Y. Nam, "The application of an integrated enterprise modeling methodologyFIDO-to supply chain integration modeling," Computers \& Industrial Engineering, vol. 4- 5 no. 1, (2003), pp.167-193.

[2] Rose C. W., "Towards a flexible deployment of business rules," Expert Systems with Applications, vol. 23 no. 4, (2002), pp.385-394.

[3] K. Bhattacharya, N. S. Caswell, Santhosh K., A. Nigam and F. Y. Wu, "Artifact-centered operational modeling Lessons learned from engagements," In: IBM SYSTEMS JOURNAL, , vol. 46 no. 4, (2007).

[4] C. E. Gerede, J. Su, "Specification and verification of artifact behaviors in business process models," Proceedings of 5th International Conference on Service-Oriented Computing (ICSOC, September, Vienna, Austria, (2007).

[5] Dong Y. W., Lou W. X. and Hao K. G., "The Software Architecture of a Business Process Management and Integration System SynchroFLOW," Computer Science, vol. 31 no. 6, (2004), pp.135-140

[6] Klaus K., "Business process modelling in the context of SOA - an empirical study of the acceptance between EPC and BPMN," World review of science, technology and sustainable development, vol. 7 no.1-2, (2010), pp.161-169.

[7] Dongen B. F., Aalst W. M. P. and Verbeek H. M. W., "Verification of EPCs using reduction rules and pe nets," Proceedings of Advanced Information Systems Engineering, Porto, Portugal, (2005), pp. 372 386

[8] Wang Z. L. and Yao Q., "Customized process modeling based on EPC,” Computer Engineering and Design, vol. 30 no. 7, (2009), pp.1652-1655.

[9] Milner R., "Communicating and mobile systems: the Pi-Calculus," Cambridge: Cambridge University Press, (1991).

[10] Feng Li-yuan, LI De-sheng, LI Wei-cui. Intelligent Process Modeling Based on -calculus . Computer Technology and Development,(2012), 22(9): pp.111-115

[11] Wang J. P., Hou G. B., Deng C. Y. and Liu Y. S., "Workflow model with extend directed graph and verification based on Pi-calculus," Computer Engineering and Design, vol. 31 no. 10, (2010), pp. 23992404.

[12] Li H. C. and Shi M. L., "Workflow Models and Their Formal Descriptions," Chinese Journal of Computers, vol. 26 no. 11, (2003), pp.1456-1463.

[13] Liao J., Tan H. and Liu J. D., "Verifying Web services substitution using Pi-calculus," Journal of Huazhong University of Science and Technology (Nature Science), vol. 33, (2005), pp. 168-171.

[14] Liao J., Tan H. and Liu J. D., "Describing and Verifying Web Service Using Pi-Calculus," Chinese Journal of Computers, vol. 28 no. 4, (2005), pp.635-643.

[15] Jia Z. C., Chen R., Zhang W. S., "Aeronautical booking process modeling for Web service and compositional compatibility verification," Computer Engineering and Applications, vol. 46 no. 24, (2010), pp.237-242. 


\section{Authors}

Wang Xia, is a teacher of school of Information Management at Shanghai Finance University (SFU), China. She received her master degree in computer application from Nanjing Hohai University. Her research interests are mainly on computer application and e-commerce.

Liu Lan, is an Associate Professor of school of Information Management at Shanghai Finance University (SFU), China. She received Ph.D. from Shanghai University of Finance and Economics. Her research interests are mainly on e-commerce and E-finance.

Man Junpeng, is a student who has graduated from SFU in July 2014. He is the leader of the national college students' innovative entrepreneurial training program from 2012 to 2013. 
International Journal of Multimedia and Ubiquitous Engineering

Vol.10, No.6 (2015) 